\title{
Hard x-ray absorption spectroscopy for pulsed sources
}

\author{
M. Kavčič, ${ }^{1}$ M. Žitnik, ${ }^{1,2}$ K. Bučar, ${ }^{1}$ A. Mihelič ${ }^{1}$ B. Marolt, ${ }^{1}$ J. Szlachetko,,${ }^{3,4}$ P. Glatzel, ${ }^{5}$ and K. Kvashnina ${ }^{5}$ \\ ${ }^{1}$ Jožef Stefan Institute, Jamova cesta 39, SI-1001 Ljubljana, Slovenia \\ ${ }^{2}$ Faculty of Mathematics and Physics, University of Ljubljana, Jadranska ulica 19, Ljubljana, Slovenia \\ ${ }^{3}$ Paul Scherrer Institut, Villigen, Switzerland \\ ${ }^{4}$ Jan Kochanowski University, Institute of Physics, Kielce, Poland \\ ${ }^{5}$ European Synchrotron Radiation Facility (ESRF), Grenoble, France
}

(Received 20 November 2012; published 6 February 2013)

\begin{abstract}
We present a method suitable for the measurement of a high-energy resolved absorption spectrum in the emission mode upon target excitation with an intense monochromatic pulse of $\mathrm{x}$-ray light. The approach is based on a measurement of a single resonant Raman x-ray spectrum at a fixed off-resonant excitation energy by a dispersive-type high-resolution emission spectrometer. Using a simple Lorentzian tail correction function and an energy-scale transformation, we reconstructed the $L_{3}$ absorption edge of Xe from the $3 d-2 p_{3 / 2}$ resonant Raman x-ray spectrum recorded at excitation energy $25 \mathrm{eV}$ below the $L_{3}$ edge. The energy resolution is well below the $L_{3}$ core-hole lifetime broadening and allows for a straightforward extraction of the $\left[2 p_{3 / 2}\right] n \ell$ resonance energies and emission strengths. The reconstructed spectrum is nearly equivalent to the high-energy resolution fluorescence detected absorption spectrum, which is recorded by scanning the photon excitation energy across the absorption edge.
\end{abstract}

DOI: 10.1103/PhysRevB.87.075106

PACS number(s): 61.05.cj, 32.30.Rj, 32.70.-n, 32.80.Fb

\section{INTRODUCTION}

X-ray absorption spectroscopy (XAS) is one of the most useful spectroscopic techniques for studying local electronic structure of materials. It is commonly implemented at the third-generation synchrotron radiation sources and has been employed extensively in various scientific fields. Often, an $\mathrm{x}$-ray absorption spectrum is recorded in the emission mode by monitoring the total intensity of the dominant radiative decay channel of the core hole while scanning the incident beam energy across the corresponding absorption edge. Eisenberger et $a{ }^{1}{ }^{1}$ were the first to report about characteristic x-ray lines narrower than the core-hole lifetime when the emitted radiation was observed with a high-resolution wavelength dispersive spectrometer. Tulkki and $\AA_{b e r g}^{2}$ gave an explanation within the framework of the resonant inelastic x-ray scattering (RIXS) and they also pointed out that the high-resolution RIXS measurements may be applied to improve spectral resolution of absorption features pertaining to the excited (bound) atomic states below the core-hole lifetime broadening. It took less than 10 years before the first absorption spectrum with a "subnatural" resolution was recorded. ${ }^{3}$ The technique of using a wavelength dispersive spectrometer to record the partial fluorescence yield across an absorption edge is nowadays referred to as the high-energy resolution fluorescence detected (HERFD) XAS. Later on, the lifetime broadening suppressed (LBS) XAS acronym was introduced for the narrowed absorption spectra deduced from a series of RIXS spectra recorded under close-to-resonant conditions. ${ }^{4,5}$

With time it became clear that a RIXS measurement may not yield a true absorption spectrum ${ }^{6,7}$ because spectral features due to the final-state multiplet splitting can not always be distinguished from spectral features arising from the intermediate excited states. ${ }^{8}$ Also, large differences between the final-state core-hole potentials may cause the measured RIXS spectra for different radiative decay channels to differ significantly from each other, ${ }^{9,10}$ and in certain cases the interference between indistinguishable absorption-emission paths affects the spectral shape. ${ }^{11,12}$ Although an accurate interpretation of RIXS spectra requires a full consideration of the inelastic resonant $\mathrm{X}$-ray scattering process, the HERFD/LBS spectrum nevertheless often provides a good approximation to the $\mathrm{x}$-ray absorption spectrum. This holds especially for the core-core transitions where the multiplet splitting due to interaction of the core hole and the promoted electron is smaller than the core-hole lifetime broadening. ${ }^{13}$ With an advent of bright thirdgeneration synchrotron sources, resonant techniques were successfully applied in a number of studies, for example, the analysis of the $K$ edges of the $3 d$ transition metals, ${ }^{14}$ the $L$ edges of the $5 d$ metals, ${ }^{15,16}$ and the absorption studies of $\mathrm{Cu}$ and Co oxides ${ }^{17,18}$ and rare-earth compounds. ${ }^{10,19,20}$

The latest development of pulsed laser, extreme ultraviolet (EUV), and x-ray sources calls for the development of ultrafast XAS, which would allow for the time-resolved studies of the local electronic structure. ${ }^{21}$ The advent of the $\mathrm{x}$-ray free electron laser (FEL) opens the possibility of performing spectroscopy in the hard $\mathrm{x}$-ray region with a femtosecond temporal resolution. Pulse-to-pulse differences due to field fluctuations as well as the problem of target radiation damage necessitate the development of a single-shot x-ray spectroscopy. In most cases, the energy band pass of a FEL pulse is not sufficient to perform single-shot XAS, and other spectroscopic techniques without the requirement of a wide incident band pass are needed. $^{22}$ X-ray emission spectroscopy (XES), which is not sensitive to the incident bandwidth and excitation energy, as long as the energy lies above the ionization threshold, could be used to probe the local electronic structure with FELs. Very recently, single-shot XES spectra dealing with the local electronic structure of Mn complexes were built up upon the target excitation with femtosecond FEL pulses, ${ }^{23}$ and excellent agreement was found with the corresponding spectra obtained by synchrotron radiation with much lower photon probe density. With availability of the self-seeded narrow bandwidth hard X-ray FEL pulses, ${ }^{24,25}$ the reconstruction 
of absorption edge by a single-pulse becomes possible. The inelastic (nonresonant) x-ray Raman scattering (XRS), which is in principle equivalent to the XAS spectrum, might be considered to perform a single-shot XAS spectroscopy. However, even for the soft X-ray absorption edges, the corresponding dipole XRS cross sections in the hard x-ray energy region are extremely small with respect to the photoabsorption cross sections $\left(\sigma_{\mathrm{XRS}} / \sigma_{\mathrm{ABS}} \sim 10^{-8}\right)$. The signal is further reduced due to a small solid angle of the Bragg crystal spectrometer so that the measurements are usually performed with multiple crystal spectrometers working in a point-to-point focusing geometry which is not applicable for the single-shot measurements. ${ }^{26,27}$ Very recently, Szlachetko et al. ${ }^{28}$ used a dispersive-type crystal spectrometer and recorded a single RIXS spectrum at a fixed off-resonant excitation energy (High Energy Resolution Off-resonant Spectroscopy - HEROS) to follow changes in the $P t(a c a c)_{2} L_{3}$ absorption spectrum with a subsecond time resolution. Even with a considerable energy detuning, the corresponding RIXS cross sections are high enough for a crystal spectrometer working in the dispersive mode ( $\sigma_{\mathrm{RIXS}} / \sigma_{\mathrm{ABS}} \sim 10^{-3}$ in the hard $\mathrm{x}$-ray energy region), which makes the off-resonant approach suitable also for the single-shot measurements.

To demonstrate the feasibility and illustrate the proposed approach in details, we report on the reconstruction of $\mathrm{Xe}$ $L_{3}$ absorption edge from a single Xe $2 p_{3 / 2}-3 d_{5 / 2}$ RIXS spectrum recorded with a dispersive $\mathrm{X}$-ray emission setup at the excitation energy $25 \mathrm{eV}$ below the $L_{3}$ edge. The achieved experimental resolution is about four times narrower than the natural lifetime broadening of the intermediate $L_{3}$ core hole, and allows a clear resolution of the strongest $\left[2 p_{3 / 2}\right] n d, n s$ resonances converging to the $L_{3}$ threshold. Accurate resonance energies and relative emission strengths are extracted upon a simple conversion of the measured RIXS spectrum. Finally, a direct equivalence with the $2 p_{3 / 2}-3 d_{5 / 2}$ HERFD $x$-ray absorption spectrum is demonstrated.

\section{EXPERIMENT}

The experiment was performed at the ID26 beamline of the European Synchrotron Radiation Facility (ESRF). The incident photon energy was tuned by means of a cryogenically cooled double-crystal monochromator. In order to maximize the energy resolution, $\mathrm{Si}(311)$ crystals were used yielding an intrinsic resolution of $\delta E / E=0.3 \times 10^{-4}$. The incident flux on the target was $\sim 10^{12}$ photons/s. The photon beam with a $50 \times 250 \mu \mathrm{m}^{2}$ cross section was directed on a stainless-steel cylindrical target cell filled with $500 \mathrm{mbar}$ of $\mathrm{Xe}$ gas and separated from the spectrometer vacuum chamber by $12.5-\mu$ m-thick Kapton windows. The target x-ray fluorescence along the polarization direction of the incident photon beam was measured with a Johansson-type in-vacuum $\mathrm{X}$-ray emission spectrometer. ${ }^{29}$ The spectrometer operated in the dispersive geometry that combines target positioning within the Rowland circle with a position-sensitive detection of diffracted $x$ rays. For gaseous targets, such off-Rowland circle geometry also enhances the efficiency since we can collect the fluorescence from the total interaction volume given by the intersection of the photon beam with the gas cell without any need to use slits to limit the size of the x-ray source. In our

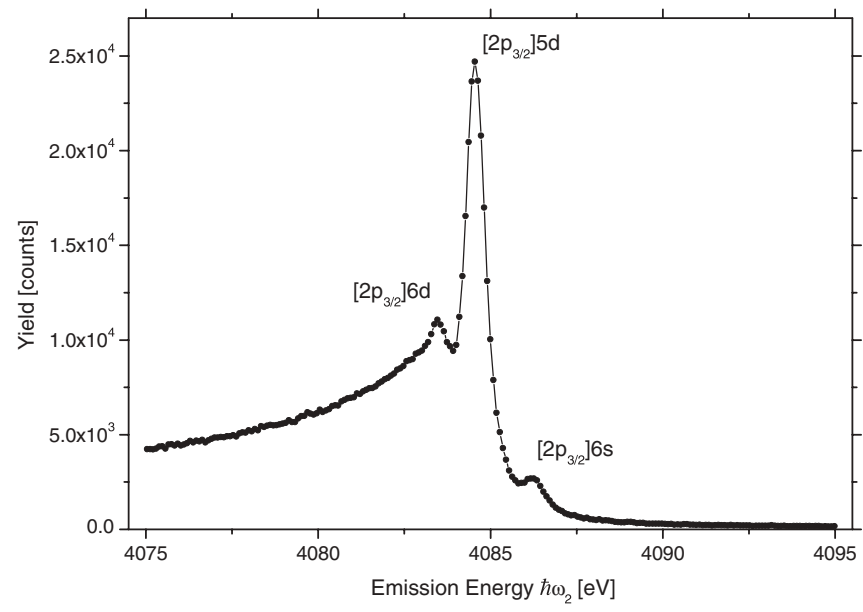

FIG. 1. The off-resonant Xe $2 p_{3 / 2}-3 d_{5 / 2}$ RIXS spectrum recorded at $4758.9 \mathrm{eV}$ excitation energy. The $\left[2 p_{3 / 2}\right] n \ell$ resonant emission signal on the high-energy side is followed by the $\left[2 p_{3 / 2}\right]$ continuum emission contribution with a characteristic Lorentzian low-energy tail.

experiment, the gas cell was placed at a distance of $42 \mathrm{~cm}$ in front of the diffraction crystal, the first-order reflection of the $\operatorname{Si}(220)$ crystal was used, and the diffracted photons were detected by a thermoelectrically cooled $\left(-40^{\circ} \mathrm{C}\right) \mathrm{CCD}$ camera consisting of $770 \times 1152$ pixels with $22.5 \times 22.5 \mu \mathrm{m}^{2}$ pixel size. The overall experimental resolution at the Xe $L_{3}$ edge was $(0.76 \pm 0.03) \mathrm{eV}$, as determined by the measurement of the full width at half maximum (FWHM) of the peak corresponding to the elastic photon scattering. The calibration of the beamline monochromator was performed by recording the elastic peak at two excitation energies around the nominal energy of $4719 \mathrm{eV}$ and compared to the energy of the characteristic $L \beta_{2}\left(L_{3}-N_{5}\right)$ emission line at $4718.86 \mathrm{eV}^{30}$ used as a reference. The latter was recorded by increasing the beam energy above the $L_{3}$ ionization threshold. The final uncertainty of this calibration procedure was $0.10 \mathrm{eV}$. First, a single RIXS spectrum was recorded at $4758.9 \mathrm{eV}$ excitation energy, about $25 \mathrm{eV}$ below the $L_{3}$ absorption edge (Fig. 1). The center of the spectrometer energy window was set to $4085 \mathrm{eV}$, about $25 \mathrm{eV}$ below the energy of the characteristic Xe $L \alpha_{1}$ line, where the RIXS signal is expected for the selected excitation energy. The total acquisition time for this spectrum was $5700 \mathrm{~s}$. The experiment was completed by the measurement of a full $2 p_{3 / 2}-3 d_{5 / 2}$ RIXS spectral map (Fig. 2). The map consists of a series of $110 \mathrm{x}$-ray emission spectra which were recorded with the spectrometer set to the energy region of the $\mathrm{Xe} L \alpha_{1}$ emission line. Each RIXS spectrum was acquired at a different value of the incident beam energy scanned across the Xe $L_{3}$ absorption edge with $0.2 \mathrm{eV}$ step, starting at $4774.0 \mathrm{eV}$ and ending at $4795.8 \mathrm{eV}$. In this measurement, the Xe pressure in the gas cell was lowered to 100 mbar to minimize the effect of self-absorption. The acquisition time for each single RIXS spectrum was $10 \mathrm{~s}$, and the count rate at excitation energy $\sim 4784 \mathrm{eV}$ (the top of $\left[2 p_{3 / 2}\right] 5 d$ resonance) was around 1000 counts/s. Two consecutive series across the absorption edge were recorded in order to check for possible leakage of the target gas cell and for general reproducibility. 


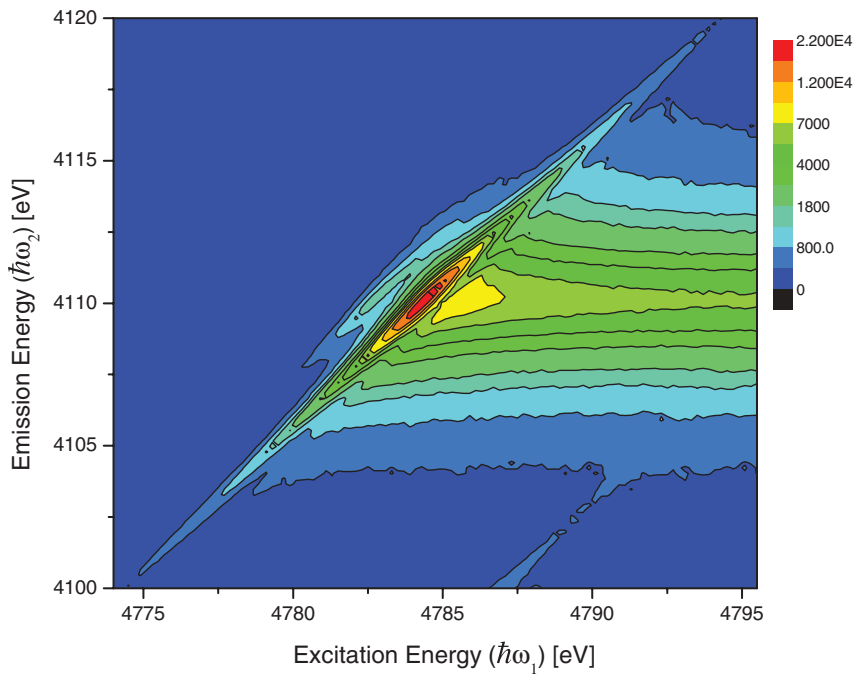

FIG. 2. (Color online) The measured Xe $2 p_{3 / 2}-3 d_{5 / 2}$ RIXS spectral map. The resonant $\left[2 p_{3 / 2}\right] n \ell$ excitations evolve along the diagonal lines characterized by a constant energy transfer $\omega_{1}-\omega_{2}$. The signal at excitation energies above the $L_{3}$ ionization threshold is dominated by the characteristic $L \alpha_{1}$ emission line.

\section{DATA ANALYSIS}

The resonant part of the general Kramers-Heisenberg equation describes RIXS differential cross section for excitation energies in the vicinity of the corresponding absorption edge. ${ }^{2}$ Neglecting the core-hole and final-state multiplet effects, the cross section is written as a sum of the resonant contribution

$$
\begin{aligned}
\frac{d \sigma_{d}}{d \omega_{2}}= & A\left(\frac{\omega_{2}}{\omega_{1}}\right) \sum_{i} \frac{E_{i f} E_{i 0}}{\left(E_{i 0}-\omega_{1}\right)^{2}+\Gamma_{i}^{2} / 4} \\
& \times g_{i f} g_{0 i} \frac{\Gamma_{f} /(2 \pi)}{\left(E_{f 0}-\omega_{1}+\omega_{2}\right)^{2}+\Gamma_{f}^{2} / 4},
\end{aligned}
$$

which describes the scattering through intermediate atomic states $|i\rangle$ to the final atomic state $|f\rangle$, and the continuum contribution which describes the scattering through the intermediate ionic state $|I\rangle$ to the final ionic state $|F\rangle$ :

$$
\begin{aligned}
\frac{d \sigma_{c}}{d \omega_{2}}= & A\left(\frac{\omega_{2}}{\omega_{1}}\right) \int_{0}^{\infty} d \varepsilon \frac{E_{I F}\left(E_{I 0}+\varepsilon\right)}{\left(E_{I 0}+\varepsilon-\omega_{1}\right)^{2}+\Gamma_{I}^{2} / 4} \\
& \times g_{I F} \frac{d g_{0 I}}{d \varepsilon} \frac{\Gamma_{F} /(2 \pi)}{\left(E_{F 0}+\varepsilon-\omega_{1}+\omega_{2}\right)^{2}+\Gamma_{F}^{2} / 4} .
\end{aligned}
$$

The incident and the scattered photon energies are denoted by $\omega_{1}$ and $\omega_{2}$. We have used the notation $E_{\mu \nu}=E_{\mu}-E_{\nu}(\mu, v=$ $0, i, I, f, F)$, where $E_{0}, E_{i}, E_{I}, E_{f}$, and $E_{F}$ are the energy of the initial (ground) state, the intermediate states, and the final states of the atom and ion, respectively. The oscillator strengths for the $0 \rightarrow i$ and $i \rightarrow f(I \rightarrow F)$ transitions are given by $g_{0 i}$ and $g_{i f}\left(g_{I F}\right)$, and $d g_{0 I} / d \varepsilon$ is the density of oscillator strength for the ejection of photoelectron with energy $\varepsilon$. The energy widths of the intermediate and final states are given by $\Gamma_{i}$ $\left(\Gamma_{I}\right)$ and $\Gamma_{f}\left(\Gamma_{F}\right)$, respectively, and $A$ is a detection angledependent scaling factor. Since the final-state energy width is much narrower than the energy width of the intermediate state, the continuum part of the RIXS cross section can be simplified by neglecting the final-state broadening. Then, the second Lorentzian factor is replaced by the Dirac delta function $\delta\left(E_{F 0}+\varepsilon-\omega_{1}+\omega_{2}\right)$ and

$$
\frac{d \sigma_{c}}{d \omega_{2}}=A\left(\frac{\omega_{2}}{\omega_{1}}\right) \frac{E_{I F}\left(E_{I F}+\omega_{1}-\omega_{2}\right)}{\left(E_{I F}-\omega_{2}\right)^{2}+\Gamma_{I}^{2} / 4} g_{I F}\left(\frac{d g_{0 I}}{d \varepsilon}\right)_{\varepsilon=\varepsilon_{p}},
$$

where $\varepsilon_{p}=\omega_{1}-\omega_{2}-E_{F 0}$. Since $d g_{0 I} / d \varepsilon$ is nonzero only for $\varepsilon>0$, the continuum contribution displays a high-energy cutoff at $\omega_{2}=\omega_{1}-E_{F 0}$.

In order to isolate the density of oscillator strength $d g_{0 I} / d \varepsilon$ representing the absorption edge, one multiplies Eq. (3) by the following correction function:

$$
f_{t}=\frac{1}{A g_{I F}}\left(\frac{\omega_{1}}{\omega_{2}}\right) \frac{\left(E_{I F}-\omega_{2}\right)^{2}+\Gamma_{I}^{2} / 4}{E_{I F}\left(E_{I F}+\omega_{1}-\omega_{2}\right)} .
$$

When the $f_{t}$ correction is applied to the whole RIXS spectrum, it will also affect the resonant RIXS contribution [Eq. (1)]. However, when the excitation energy is fixed far below the ionization threshold at $\omega_{1}=E_{I 0}-\Delta$, where $\Delta \gg$ $\Gamma_{i}, E_{i I}, E_{f F}$, the correction function is changing relatively slowly with $\omega_{2}$, and its behavior is mainly determined by the inverse of the Lorentzian tail of the characteristic emission line. In this case, the effect of the correction function can be closely reproduced by simply multiplying the contribution of resonance $|i\rangle$ with the correction function $f_{t}$ evaluated at the emission energy corresponding to the center of the resonance $\left(\omega_{2}=\omega_{1}-E_{f 0}=E_{I f}-\Delta\right)$, so that

$$
\begin{aligned}
f_{t} & \frac{d \sigma_{d}}{d \omega_{2}} \\
\approx & \sum_{i}\left[\left(\frac{g_{i f}}{g_{I F}}\right) \frac{\left(E_{f F}+\Delta\right)^{2}+\Gamma_{I}^{2} / 4}{\left(E_{i I}+\Delta\right)^{2}+\Gamma_{i}^{2} / 4} \frac{E_{i f} E_{i 0} g_{0 i}}{E_{I F}\left(E_{I F}+E_{f 0}\right)}\right. \\
& \left.\times \frac{\Gamma_{f} /(2 \pi)}{\left(E_{f 0}-\omega_{1}+\omega_{2}\right)^{2}+\Gamma_{f}^{2} / 4}\right] \\
\approx & \sum_{i} g_{0 i} \frac{\Gamma_{f} /(2 \pi)}{\left(E_{f 0}-\omega_{1}+\omega_{2}\right)^{2}+\Gamma_{f}^{2} / 4}
\end{aligned}
$$

We have assumed that for the inner-shell radiative transition, the oscillator strengths $g_{i f}$ and $g_{I F}$ as well as the energy widths involved are equal and the weak dependence of prefactors on $\omega_{1}$ or $\omega_{2}$ in a relatively narrow energy interval of interest was neglected. Similarly to the continuum part, multiplying the resonant RIXS contribution by $f_{t}$ isolates a resonant part of the absorption spectrum. In order to reach the absorption spectrum, the RIXS spectral intensity $I_{\text {RIXS }}^{\omega_{1}}\left(\omega_{2}\right)$ recorded at the fixed excitation energy $\omega_{1}$ well below the absorption edge should be multiplied by an inverse Lorentzian tail correction function yielding

$$
\begin{aligned}
& I_{\mathrm{HEROS}}^{\omega_{1}}\left(\omega_{2}\right) \\
& \quad=I_{\mathrm{RIXS}}^{\omega_{1}}\left(\omega_{2}\right)\left[\left(E_{I F}-\omega_{2}\right)^{2}+\Gamma_{I}^{2} / 4\right] \\
& \quad \propto \sum_{i} g_{0 i} \frac{\Gamma_{f} /(2 \pi)}{\left(\omega_{1}-\omega_{2}-E_{f 0}\right)^{2}+\Gamma_{f}^{2} / 4}+\left(\frac{d g_{0 I}}{d \varepsilon}\right)_{\varepsilon=\varepsilon_{p}} .
\end{aligned}
$$

In agreement with a recent suggestion, ${ }^{28}$ the resulting spectrum is given an acronym HEROS, because it is derived from the RIXS spectrum at a fixed off-resonant excitation. Similar to 


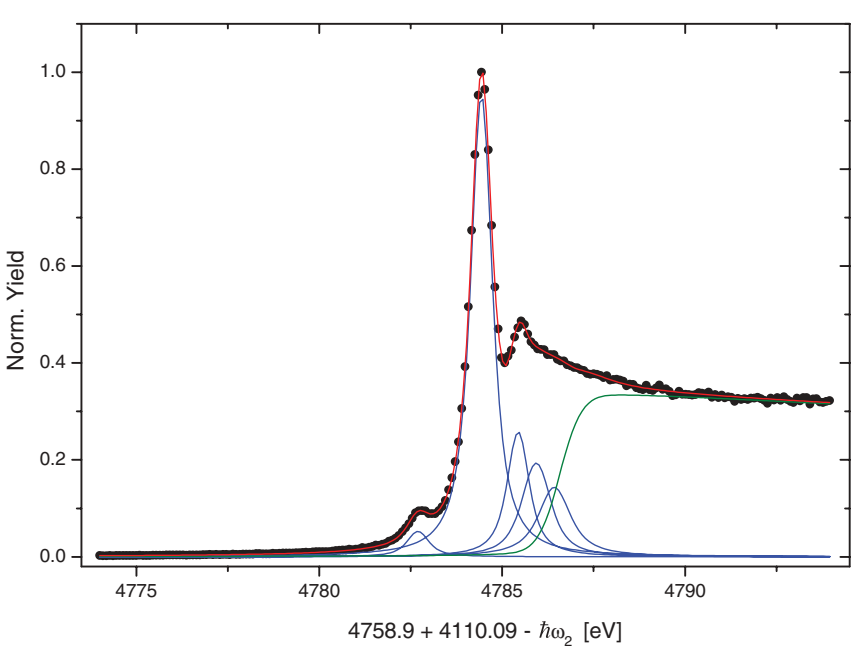

FIG. 3. (Color online) The HEROS $L_{3}$ absorption spectrum of Xe extracted from the measured $2 p_{3 / 2}-3 d_{5 / 2}$ RIXS spectrum, which is recorded at $4758.9 \mathrm{eV}$ excitation energy. The spectrum is fitted with a sum of Voigt functions describing the $\left[2 p_{3 / 2}\right] n d$ and $n s$ discrete excitations followed by a step function corresponding to the $\left[2 p_{3 / 2}\right]$ absorption edge.

HERFD, a HEROS spectrum also corresponds to an absorption spectrum with spectral resolution defined by the (narrow) final-state broadening. While HERFD directly yields the resonance energies $E_{i 0}$, the absolute energies of the resonant contributions in the HEROS spectrum depend on the excitation energy and are ordered in the mirrored direction (energetically lower bound states resonate at higher emission energies). According to Eq. (6), the HEROS resonance emission energy is

$$
\omega_{2}=\omega_{1}-E_{f 0}=\omega_{1}+E_{i f}-E_{i 0} .
$$

The energy difference $E_{i f} \approx E_{I F}$ corresponds to the characteristic emission energy of the $i \rightarrow f$ transition. The absolute resonance energies $E_{i 0}$ can thus be determined directly from the measured HEROS spectrum by plotting the intensity as a function of $\omega_{1}+E_{I F}-\omega_{2}$ instead of $\omega_{2}$. After this transformation has been made, the HEROS spectrum is placed on the energy scale of the absorption spectrum and comes into direct correspondence with the HERFD spectrum.

\section{RESULTS AND DISCUSSION}

The Xe $L_{3}$ HEROS absorption spectrum was obtained from the $2 p_{3 / 2}-3 d_{5 / 2}$ RIXS spectrum recorded at the excitation energy $\omega_{1}=4758.9 \mathrm{eV}$ by multiplication with the tail correction function given by Eq. (6). In this procedure, the $L \alpha_{1}$ emission energy $E_{I F}$ was taken to be $4110.09 \mathrm{eV}^{31}$ and the energy width of the intermediate $2 p_{3 / 2}$ core-hole states was set to $2.88 \mathrm{eV}$. The latter was determined by the analysis of the $\left[2 p_{3 / 2}\right] 5 d$ resonance yield isolated in the measured $2 p_{3 / 2}-3 d_{5 / 2}$ RIXS spectral map (Fig. 2). The $I_{\text {HEROS }}^{\omega_{1}}$ spectrum plotted as a function of $\left(8868.99 \mathrm{eV}-\omega_{2}\right)$ is presented in Fig. 3. In order to extract energies and relative intensities pertaining to different excitations, we have fitted the spectrum with a sum of Voigt functions describing resonant contributions followed by a linear combination of Gaussian and Lorentzian cumulative

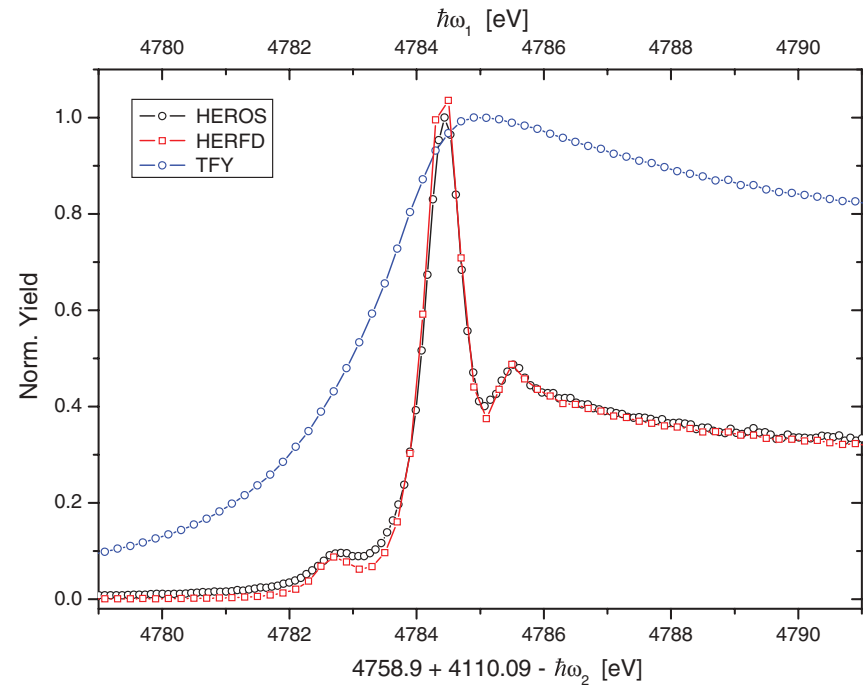

FIG. 4. (Color online) The HEROS $L_{3}$ absorption spectrum reconstructed from the $2 p_{3 / 2}-3 d_{5 / 2}$ RIXS spectrum recorded at $4758.9 \mathrm{eV}$ excitation energy compared with the $2 p_{3 / 2}-3 d_{5 / 2}$ HERFD spectrum taken at $4110.09 \mathrm{eV}$ emission energy. The total fluorescence yield (TFY) spectrum is also presented for comparison.

distribution, which is used to describe the absorption edge. The extracted energies and intensities are reported in Table I.

The previously published experimental values are based on the standard photoabsorption measurements, ${ }^{32,33}$ which are affected by the large $L_{3}$ hole lifetime broadening and yield a smooth absorption profile with no pronounced structures. This resulted in a very limited precision of the extracted resonance energies (especially for the weak [2 $\left.2 p_{3 / 2}\right] 6 s$ state) without any relative intensities reported. This was improved recently by recording a series of RIXS spectra across the $L_{3}$ absorption edge. An elaborated fitting procedure based on the parametrization of the RIXS cross section was used to assess the energies and emission strengths of the discrete resonances below the $L_{3}$ edge in $\mathrm{Xe}^{34}$ The method presented in this work provides a narrowed $\mathrm{Xe} L_{3}$ absorption spectrum without scanning the excitation energy across the absorption edge, and yields accurate spectroscopic information on the excitations to the $\left[2 p_{3 / 2}\right] n \ell$ states without the need for detailed theoretical modeling.

Having also recorded the full $2 p_{3 / 2}-3 d_{5 / 2}$ RIXS spectral map of Xe (Fig. 2), the equivalence between the HERFD and HEROS absorption spectra can be verified experimentally. In our case, the HERFD $2 p_{3 / 2}-3 d_{5 / 2}$ spectrum of Xe was obtained from a horizontal cut through the measured RIXS plane at the emission energy $\omega_{2}=4110.09 \mathrm{eV}$ corresponding to the energy of the $2 p_{3 / 2}-3 d_{5 / 2}$ transition (the top of the $L \alpha_{1}$ line). The two spectra $I_{\text {HERFD }}^{\omega_{2}}\left(\omega_{1}\right)$ and $I_{\text {HEROS }}^{\omega_{1}}\left(8868.99 \mathrm{eV}-\omega_{2}\right)$, are presented in Fig. 4 together with the total fluorescence yield (TFY) spectrum. The latter was obtained by projecting the total signal in the RIXS map on the excitation energy axis. While the TFY spectrum shows a smooth profile due to the $L_{3}$ lifetime broadening, drastic narrowing is observed in both resonant spectra, that exposes separate $\left[2 p_{3 / 2}\right] n \ell$ resonant excitations. The HEROS absorption spectrum derived from a single off-resonant experimental RIXS spectrum matches the recorded HERFD spectrum almost perfectly. However, 
TABLE I. The energy differences $E_{i 0}$ and relative emission strengths $f_{0 i f}$ for population of the $\left[3 d_{5 / 2}\right] n \ell$ final states extracted from the fit of the HEROS absorption spectrum (bold numbers) compared with previously published data. The error estimates of the fitting procedure are given in parentheses. The uncertainty of the energy calibration is $0.10 \mathrm{eV}$.

\begin{tabular}{|c|c|c|c|c|c|c|c|}
\hline \multicolumn{3}{|c|}{$E_{i}-E_{0}$} & \multicolumn{2}{|l|}{ Others $(\mathrm{eV})$} & $|f\rangle$ & $f_{0 i f}(0 \rightarrow i \rightarrow f)$ & Others \\
\hline$\left[2 p_{3 / 2}\right] 6 s$ & $4782.70(1)$ & $4782.40^{32}$ & & $4782.97(3)^{34}$ & {$\left[3 d_{5 / 2}\right] 6 s$} & $0.055(2)$ & $0.061(4)^{34}$ \\
\hline$\left[2 p_{3 / 2}\right] 5 d$ & 4784.43(1) & $4784.23^{32}$ & $4784.3^{33}$ & $4784.72(1)^{34}$ & {$\left[3 d_{5 / 2}\right] 5 d$} & $1.00(2)$ & $1.000(3)^{34}$ \\
\hline$\left[2 p_{3 / 2}\right] 6 d$ & $4785.43(3)$ & $4785.26^{32}$ & & $4785.74(1)^{34}$ & {$\left[3 d_{5 / 2}\right] 6 d$} & $0.27(6)$ & $0.244(2)^{34}$ \\
\hline$\left[2 p_{3 / 2}\right] 7 d$ & 4785.93(31) & $4785.70^{32}$ & & & & & \\
\hline$|I\rangle$ & $E_{I}-E_{0}$ & & & & & & \\
\hline$\left[2 p_{3 / 2}\right]$ & 4786.59(35) & $4786.31^{32}$ & $4785.2^{33}$ & $4786.67(2)^{34}$ & & & \\
\hline
\end{tabular}

the width and also the spectral shape are not completely identical for both, as particularly evident close to the weak $\left[2 p_{3 / 2}\right] 6 s$ resonance situated on the tail of the dominant $\left[2 p_{3 / 2}\right] 5 d$ signal. The shape of the resonant contribution in the HEROS spectrum is expressed by a single Lorentzian with the final-state broadening $\Gamma_{f}$. On the other hand, the shape of the HERFD spectrum corresponding to a constant emission scan at $\omega_{2}=E_{I F} \approx E_{i f}$ is given by a product of two Lorentzians with the widths equal to $\Gamma_{f}$ and $\Gamma_{i}$. When $\Gamma_{f} \ll \Gamma_{i}$, the HERFD spectral shape is mainly governed by the narrower Lorentzian, and the effective broadening practically equals $\Gamma_{f}$. In principle, however, the final HERFD line broadening is slightly smaller than $\Gamma_{f}$, as observed also by comparing the two spectra presented in Fig. 4. The expected small difference might be further enhanced due to significant differences between the spectrometer and photon probe resolution.

The equivalence of HEROS and HERFD absorption spectra is easily understood by looking at the RIXS plane in Fig. 2. When a constant emission scan (horizontal cut through the RIXS plane) is performed, we are moving through the consecutive narrow discrete resonant features, each with its own specific energy transfer. The same information should be obtained by performing a fixed excitation scan (vertical cut through the RIXS plane), provided that the excitation energy is well below the absorption edge to avoid a resonant enhancement of any particular excitation. In both cases, we are actually measuring the intensity of the scattered photons as a function of the photon energy transfer $\omega_{1}-\omega_{2}$ by changing either of the two energies while keeping the other one fixed. However, there is an important conceptual difference between the two methods. As shown above, in the vertical (fixed excitation) cut, the dispersion of the energy transfer is achieved by the spectrometer, so that the high-resolution spectrum can be obtained even without a tunable x-ray source. In combination with a dispersive-type emission spectrometer, the scanning is completely avoided. Such an approach enables shot-by-shot measurements and in principle allows acquisition of a full spectrum by a single intense monochromatic $\mathrm{x}$-ray pulse.

\section{CONCLUSIONS}

We have demonstrated that the same spectroscopic information can be extracted either from a HERFD spectrum relying on dispersion in the excitation energy, or from a single off-resonant RIXS spectrum, relying on dispersion in the emission energy. In the latter case, high-resolution spectroscopy of the absorption edge could be performed also with a fixed-energy laboratory $\mathrm{x}$-ray source. The approach with fixed excitation energy could also be combined with another $\mathrm{x}$-ray technique, for example, x-ray diffraction. Because of scanning-free arrangement, the in situ absorption studies with subsecond time resolution can be performed. ${ }^{28}$ Finally, the HEROS spectroscopy is suitable for XAS with intense single-shot target excitation that could be used with hard $\mathrm{X}$-ray FEL sources. The main obstacles for this technique are low RIXS cross sections for excitation energies well below the resonances. In our experiment with photon flux of $\sim 10^{12}$ photons/s, a maximum count rate of $\sim 4$ counts $/ \mathrm{s}$ was observed at the top of the main spectral line corresponding to the $\left[2 p_{3 / 2}\right] 5 d$ excitation. Assuming $10^{12}$ photons in a single FEL hard $x$-ray pulse, the observed count rate would roughly correspond to the number of expected counts per pulse. Solely by performing the experiment at a two times smaller energy offset, about a factor of 4 in intensity would be gained without a considerable spectral distortion. However, with solid targets and dedicated high-efficiency emission spectrometers, ${ }^{35}$ the count rate could still significantly increase. A single-shot hard x-ray absorption spectroscopy at the FEL sources could therefore be feasible using the method described in this work.

\section{ACKNOWLEDGMENTS}

An excellent assistance of the ID26 beam line staff with the preparation of the experiment is acknowledged. This work is supported by the Slovenian Ministry of Education, Science and Technology through the research program P1-0112.
${ }^{1}$ P. Eisenberger, P. M. Platzman, and H. Winick, Phys. Rev. Lett. 36, 623 (1976).

${ }^{2}$ J. Tulkki and T. Åberg, J. Phys. B: At., Mol. Opt. Phys. 15, L435 (1982).
${ }^{3}$ K. Hämäläinen, D. P. Siddons, J. B. Hastings, and L. E. Berman, Phys. Rev. Lett. 67, 2850 (1991).

${ }^{4}$ H. Hayashi, Y. Udagawa, W. A. Caliebe, and C. C. Kao, Chem. Phys. Lett. 371, 125 (2003). 
${ }^{5}$ H. Hayashi, Anal. Sci. 24, 15 (2008).

${ }^{6}$ P. Carra, M. Fabrizio, and B. T. Thole, Phys. Rev. Lett. 74, 3700 (1995).

${ }^{7}$ P. W. Loeffen, R. F. Pettifer, S. Müllender, M. A. van Veenendaal, J. Röhler, and D. S. Silvia, Phys. Rev. B 54, 14877 (1996).

${ }^{8}$ P. Glatzel, M. Sikora, and M. Fernández-García, Eur. Phys. J. Special Topics 169, 207 (2009).

${ }^{9}$ A. Kotani, K. O. Kvashnina, S. M. Butorin, and P. Glatzel, J. Electron Spectrosc. Relat. Phenom. 184, 210 (2011).

${ }^{10}$ A. Kotani, K. O. Kvashnina, P. Glatzel, J. C. Parlebas, and G. Schmerber, Phys. Rev. Lett. 108, 036403 (2012).

${ }^{11}$ G. Vankó, F. M. F. de Groot, S. Huotari, R. J. Cava, T. Lorenz, and M. Reuther, arXiv:0802.2744.

${ }^{12}$ M. Kavčič, M. Žitnik, K. Bučar, A. Mihelič, S. Carniato, L. Journel, R. Guillemin, and M. Simon, Phys. Rev. Lett. 105, 113004 (2010).

${ }^{13}$ P. Glatzel, T.-Ch. Weng, K. Kvashnina, J. Swarbrick, M. Sikora, E. Gallo, N. Smolentsev, and R. Alonso Mori, J. Electron Spectrosc. Relat. Phenom. (2012), doi: 10.1016/j.elspec.2012.09.004.

${ }^{14}$ P. Glatzel and U. Bergmann, Coord. Chem. Rev. 249, 65 (2005).

${ }^{15}$ F. M. F. de Groot, M. H. Krisch, and J. Vogel, Phys. Rev. B 66, 195112 (2002).

${ }^{16}$ J. A. van Bokhoven, C. Louis, J. T. Miller, M. Tromp, O. V. Safonova, and P. Glatzel, Angew. Chem. 45, 4651 (2006).

${ }^{17}$ H. Hayashi, R. Takeda, Y. Udagawa, T. Nakamura, H. Miyagawa, H. Shoji, S. Nanao, and N. Kawamura, Phys. Rev. B 68, 045122 (2003).

${ }^{18}$ G. Dräger and P. Machek, Phys. Rev. B 79, 033103 (2009).

${ }^{19}$ H. Hayashi, R. Takeda, M. Kawata, Y. Udagawa, N. Kawamura, Y. Watanabe, and S. Nanao, Phys. Rev. B 70, 155113 (2004).

${ }^{20}$ H. Hayashi, M. Kawata, A. Sato, Y. Udagawa, T. Inami, K. Ishii, H. Ogasawara, and S. Nanao, Phys. Rev. B 72, 045114 (2005).
${ }^{21}$ C. Bressler and M. Chergui, Chem. Rev. 104, 1781 (2004).

${ }^{22} \mathrm{U}$. Bergmann, http://www-ssrl.slac.stanford.edu/lcls/sac/saconly/ index.html

${ }^{23}$ R. Alonso-Mori et al., Proc. Natl. Acad. Sci. USA (2012), doi: 10.1073/pnas.1211384109.

${ }^{24}$ G. Geloni, V. Kocharyan, and E. Saldin, arXiv:1012.3617.

${ }^{25}$ J. Amann et al., Nat. Photonics 6, 693 (2012).

${ }^{26}$ D. T. Bowron, M. H. Krisch, A. C. Barnes, J. L. Finney, A. Kaprolat, and M. Lorenzen, Phys. Rev. B 62, R9223 (2000).

${ }^{27}$ D. Sokaras, D. Nordlund, T.-C. Weng, R. Alonso Mori, P. Velikov, D. Wenger, A. Garachtchenko, M. George, V. Borzenets, B. Johnson, Q. Qian, T. Rabedeau, and U. Bergmann, Rev. Sci. Instrum. 83, 043112 (2012).

${ }^{28}$ J. Szlachetko, M. Nachtegaal, J. Sa, J.-Cl. Dousse, J. Hoszowska, E. Kleymenov, M. Janousch, O. V. Safonova, C. König, and J. A. van Bokhoven, Chem. Commun. 48, 10898 (2012).

${ }^{29}$ M. Kavčič, M. Budnar, A. Mühleisen, F. Gasser, M. Žitnik, K. Bučar, and R. Bohinc, Rev. Sci. Instrum. 83, 033113 (2012).

${ }^{30}$ M. Ohno and R. E. LaVilla, Phys. Rev. A 45, 4713 (1992).

${ }^{31}$ R. Deslattes, E. G. Kessler Jr., P. Indelicato, L. de Billy, E. Lindroth, and J. Anton, Rev. Mod. Phys. 75, 35 (2003).

${ }^{32}$ M. Breinig, M. H. Chen, G. E. Ice, F. Parente, B. Crasemann, and G. S. Brown, Phys. Rev. A 22, 520 (1980).

${ }^{33}$ A. Kodre, J. Padežnik Gomilšek, I. Arčon, and G. Aquilanti, Phys. Rev. A 82, 022513 (2010).

${ }^{34}$ M. Žitnik, M. Kavčič, K. Bučar, A. Mihelič, M. Štuhec, J. Kokalj, and J. Szlachetko, Phys. Rev. A 76, 032506 (2007).

${ }^{35}$ R. Alonso-Mori, J. Kern, D. Sokaras, T.-Ch. Weng, D. Nordlund, R. Tran, P. Montanez, J. Delor, V. K. Yachandra, J. Yano, and U. Bergmann, Rev. Sci. Instrum. 83, 073114 (2012). 